\title{
The Role of $p$-Oligosiloxane Substituents of Polystyrene in Selective Oxygen Permeation through the Polymer Film
}

\author{
Yuhsuke KAWAKAMI, * Hiroo KaRASAWA, Hiroki KAMIYA, \\ Toshiki AOKI, and Yuya YAMASHITA \\ Department of Synthetic Chemistry, Faculty of Engineering, Nagoya University, \\ Chikusa, Nagoya 464, Japan
}

(Received July 1, 1985)

\begin{abstract}
Selective oxygen permeation through the films of $p$-(oligosiloxanylstyrene)s was investigated, and the role of the oligosiloxane substituents is discussed based on a dissolutiondiffusion mechanism. The oligosiloxane side chains were found to play an important role to enhance the diffusion of oxygen.

KEY WORDS Oligosiloxane / Macromer / Film / Oxygen Permeation / Mechanism / Dissolution-Diffusion / Activation Energy / Free Volume /
\end{abstract}

Poly( $p$-oligosiloxanylstyrene)s were reported to be good self-supporting oxygen permeable membrane materials. ${ }^{1,2}$ In that report, an imaginative structure of polymer film was postulated, ${ }^{2}$ in which oxygen permeation is influenced by both polymer back bones and oligosiloxane side chains. In order to obtain more understanding of the permeation, the permeation behavior of air through the films of poly( $p$-oligosiloxanylstyrene)s were analyzed based on dissolution-diffusion mechanism.

\section{EXPERIMENTAL}

\section{Monomer}

1-(p-Bromophenyl)-1-butanone. This compound was synthesized similarly to the reported method, ${ }^{3}$ and the solid product was recrystallized from petroleum ether. $90 \%$ Yield. mp $37-39^{\circ} \mathrm{C}$.

4- $n$-Butyl-1-bromobenzene 1-(4-Bromophenyl)-1-butanone was reduced by hydrazine to give the product. $^{4,5}$

4-n-Butylstyrene

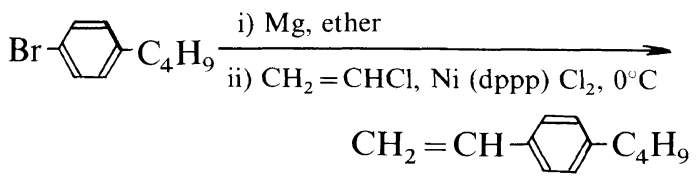

Vinylchloride $(248 \mathrm{mmol})$ and Nickel chloride-bis(diphenylphosphinopropane) complex $\left[\mathrm{Ni}(\mathrm{dppp}) \mathrm{Cl}_{2}\right](40 \mathrm{mg})$ were added in ether $(100 \mathrm{ml})$, and small amounts of methylmagnesium bromide were slowly added until all $\mathrm{Ni}(\mathrm{dppp}) \mathrm{Cl}_{2}$ completely dissolved. To this reaction mixture, 4- $n$-butylphenylmagnesium bromide prepared from activated $\mathrm{Mg}$ turnings $(250 \mathrm{mmol})$ by dibromoethane $(0.6 \mathrm{ml})$ and 4- $n$-butyl-1-bromobenzene $(200 \mathrm{mmol})$ in ether $(100 \mathrm{ml})$ was added drop wise during $1 \mathrm{~h}^{6}$ After the addition, the mixture was stirred overnight, decomposed with aq $\mathrm{NH}_{4} \mathrm{Cl}$, and extracted with ether. Distillation gave the product in $75 \%$ yield. The compound was further purified by silica gel column chromatography in order to remove the uncoupled product, butylbenzene. ${ }^{1} \mathrm{H}$ NMR $\left(\mathrm{CDCl}_{3}\right) \delta$ $0.92\left(\mathrm{t}, 3 \mathrm{H}, J=7.0 \mathrm{~Hz}, \mathrm{C}_{3}\right), 0.9-2.07(\mathrm{~m}$, $\left.6 \mathrm{H},\left(\mathrm{C}_{2}\right)_{3}\right), 2.57(\mathrm{t}, 2 \mathrm{H}, J=7.0 \mathrm{~Hz}, \quad \nabla-$ $\left.\mathrm{CH}_{2}\right), 5.07\left(\mathrm{q}, 1 \mathrm{H}, J_{1}=1.0 \mathrm{~Hz}, J_{2}=7.0 \mathrm{~Hz}\right.$, 
$\underline{\mathrm{H}}=), \quad 5.77 \quad\left(\mathrm{q}, \quad 1 \mathrm{H}, \quad J_{1}=1.0 \mathrm{~Hz}, \quad J_{2}=\right.$ $\left.10.0 \mathrm{~Hz}, \underline{\mathrm{H}}^{-\overline{\mathrm{C}}}\right), 6.62\left(\mathrm{q}, 1 \mathrm{H}, J_{1}=7.0 \mathrm{~Hz}\right.$, $\left.J_{2}=10.0 \mathrm{~Hz}, \quad \quad \mathrm{H}^{-}\right), \quad 6.97$ and $7.25 \mathrm{ppm}$

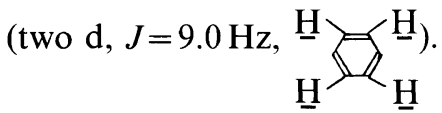

All other monomers used in this study were prepared as reported. ${ }^{2}$ The characteristics of the polymers are shown in Table I.

Films were prepared by casting technique from THF solution by evaporating the solvent during $24-48 \mathrm{~h}$. The films were detached from the slide glass by immersing into water and dried in vacuum for $24 \mathrm{~h}$, and conditioned at $35^{\circ} \mathrm{C}$ under vacuum for $24 \mathrm{~h}$. The thickness of the films were in the order of $80-100 \mu \mathrm{m}$. The permeation behavior from upstream at atmospheric pressure to downstream in vacuum was estimated by gas chromatographic method for supplied air at various temperatures.

\section{Treatment of the Data}

Typical changes in the amounts of permeated gases are shown in Figure 1.

The obtained data were treated based on Fick's and Henry's laws.

$$
q=\mathrm{DSp}_{1} A(t-\theta) / l
$$

where, $D$ and $S$ are diffusion and solubility coefficients, and $p_{1}$ is the partial pressure of the permeate at upstream side (pressure of down stream side is approximated to be 0 ), $A$ is the area of permeation, $l$, thickness of the film, $t$, time, and $\theta$, time lag in permeation.

The permeation coefficient is defined by following equation.

$$
P=D S
$$

The diffusion coefficient can be estimated from the time lag in permeation.

$$
D=l^{2} / 6 \theta
$$

By measuring $q, p_{1}, A, l$, and $\theta, P$ and $D$ were estimated by the least square method, and $S$ was calculated from $P$ and $D .^{7-9}$
Table I. Characteristics of polymers in this study"

\begin{tabular}{lcrc}
\hline Polymer & $M_{w} \times 10^{4}$ & $M_{n} \times 10^{4}$ & $T_{\mathrm{g}} / \mathrm{C}$ \\
\hline PolyS1 & 21.7 & 9.0 & 136 \\
PolyS2 & 23.8 & 8.4 & 36 \\
PolyS3 & 78.1 & 36.5 & $-10<$ \\
PolyS5 & 34.9 & 9.6 & $-30<$ \\
PolyB1 & 83.0 & 46.5 & 85 \\
PolyB2 & 56.4 & 21.0 & -3 \\
PolySt4 & 14.0 & 4.3 & 114 \\
PolyBS & 47.1 & 18.3 & 130 \\
PolySt & 33.3 & 20.5 & 108 \\
\hline
\end{tabular}

"PolySn indicates a polymer from a monomer of the type

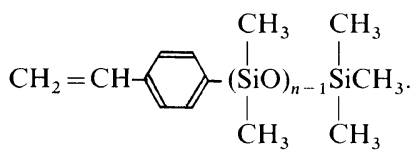

PolyBn indicates a polymer from a monomer of the type

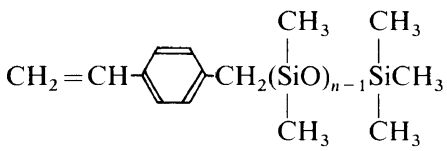

PolySt4, polyBS, and polySt are poly $[p$-tris(trimethylsiloxy)silylstyrene], poly( $p$ - $n$-butylstyrene), and polystyrene, respecitvely.

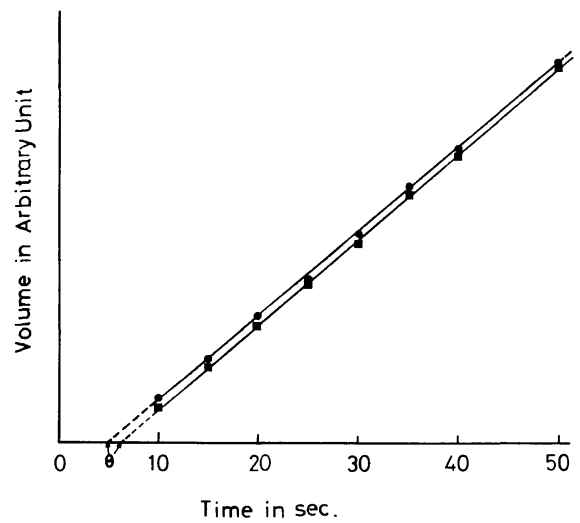

Figure 1. Typical permeation behavior of oxygen and Nitrogen through polyS 2 Film at $25^{\circ} \mathrm{C}$ : oxygen; nitrogen.

Selectivity in permeation is defined by eq 4 .

$$
\alpha=P_{\mathrm{O}_{2}} / P_{\mathrm{N}_{2}}
$$

The activation energy in permeation was 
Table II. Permeation behavior of polymers

\begin{tabular}{|c|c|c|c|c|c|c|c|c|}
\hline Polymer & $P_{\mathrm{O}_{2}}{ }^{a}$ & $\alpha$ & $S_{\mathrm{O}_{2}}{ }^{\mathrm{b}}$ & $S_{\mathrm{N}_{2}}{ }^{\mathrm{b}}$ & $D_{\mathrm{O}_{2}}{ }^{c}$ & $D_{\mathrm{N}_{2}}{ }^{\mathrm{c}}$ & $E_{\mathrm{P}, \mathrm{O}_{2}}{ }^{\mathrm{d}}$ & $E_{\mathrm{P}, \mathrm{N}_{2}}{ }^{\mathrm{d}}$ \\
\hline PolySt & $\begin{array}{c}1.2 \\
(1)^{\mathrm{e}}\end{array}$ & 5.5 & 2.2 & 1.1 & $\begin{array}{r}0.56 \\
(1)^{\mathrm{e}}\end{array}$ & 0.2 & 3.0 & 4.3 \\
\hline PolyBS & $\begin{array}{c}6.7 \\
(5.6)^{\mathrm{e}}\end{array}$ & 3.2 & 1.0 & - & $\begin{array}{r}6.7 \\
(12)^{\mathrm{e}}\end{array}$ & - & 5.1 & 6.8 \\
\hline PolyS2 & $\begin{array}{l}40 \\
(33)^{\mathrm{e}}\end{array}$ & 3.0 & 2.2 & 1.3 & $\begin{array}{c}18 \\
(32)^{\mathrm{e}}\end{array}$ & 11 & $\begin{array}{l}3.2\left(T_{\mathrm{g}}>T\right) \\
4.0\left(T_{\mathrm{g}}<T\right)\end{array}$ & $\begin{array}{l}4.4\left(T_{\mathrm{g}}>T\right) \\
5.5\left(T_{\mathrm{g}}<T\right)\end{array}$ \\
\hline PolyB1 & $\begin{array}{c}14 \\
(12)^{\mathrm{e}}\end{array}$ & 3.9 & 2.1 & 1.1 & $\begin{array}{r}6.5 \\
(12)^{\mathrm{e}}\end{array}$ & 3.3 & 1.9 & 3.0 \\
\hline PolyB2 & $\begin{array}{l}40 \\
(33)^{\mathrm{e}}\end{array}$ & 3.0 & 2.5 & 1.2 & $\begin{array}{c}16 \\
(29)^{\mathrm{e}}\end{array}$ & 11.3 & 7.0 & 8.9 \\
\hline PolyE ${ }^{10)}$ & 2.9 & 3.0 & 0.63 & 0.3 & 4.6 & 3.2 & 10.1 & 11.8 \\
\hline $\mathrm{PDMS}^{11) \mathrm{f}}$ & $489^{g}$ & $2.2^{\mathrm{g}}$ & $4.1^{\mathrm{g}}$ & 2.7 & $120^{\mathrm{g}}$ & 85 & $2.7^{\mathrm{g}, \mathrm{h}}$ & $2.4^{\mathrm{g}, \mathrm{h}}$ \\
\hline 12) & 352 & 1.9 & 1.9 & 1.5 & 189 & 123 & - & - \\
\hline
\end{tabular}

The values of $P_{\mathrm{O}_{2}}, D_{\mathrm{O}_{2}}$, and $S_{\mathrm{O}_{2}}$ are those at $25^{\circ} \mathrm{C}$.

a In $10^{-10} \mathrm{~cm}^{3}$ (STP) $\mathrm{cm} \mathrm{cm}^{-2} \mathrm{~s}^{-1} \mathrm{cmHg}^{-1}$ unit.

b In $10^{-3} \mathrm{~cm}^{3}$ (STP) $\mathrm{cm}^{-3} \mathrm{~cm} \mathrm{Hg}^{-1}$ unit.

c In $10^{-7} \mathrm{~cm}^{2} \mathrm{~s}^{-1}$ unit.

d In kcal mol ${ }^{-1}$ unit.

e Relative value to polySt.

f PDMS is polydimethylsiloxane.

g Values at $0{ }^{\circ} \mathrm{C}$ for $10 \%$ cross-linked sample.

h Values of $E_{\mathrm{D}}$.

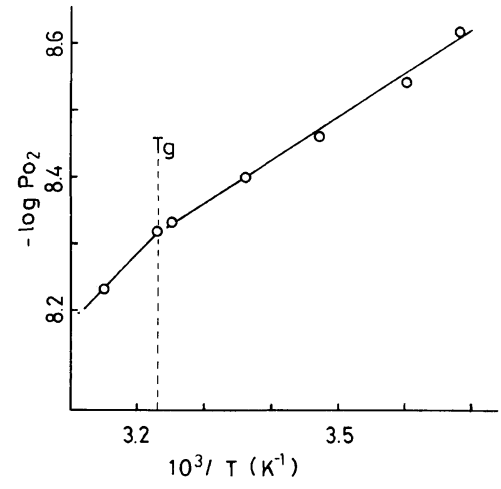

Figure 2. Typical arrhenius plot of the permeation of Oxygen through polyS2 film.

evaluated from an Arrhenius plot. An example of such a plot is shown in Figure 2.

$$
P=P^{*} \exp \left(-E_{\mathrm{p}} / R T\right)
$$

\section{RESULTS AND DISCUSSION}

We have been interested in designing good oxygen permeable membrane materials by introducing short dimethylsiloxane branches into the polymer back bone which has relatively high $T_{\mathrm{g}}$. By this approach, polyS2 was found to be a good self-supporting selective oxygen permeable membrane material. This was thought to be because these short dimethylsiloxane branched polymers do not phaseseparate, and the gases permeate through non phase-separated membrane under the influence of polymer back bone component. ${ }^{2}$

The results of permeation are shown in Table II.

PolyS2 and polyB2 show high permeability coefficients and good selectivity in permeation. Especially, polyS2 has self-supporting property and is a good candidate for a material of selective oxygen permeable membrane. PolyB2 does not have enough self-supporting property, but copolymers of B2 will also be good materials for oxygen permeable membranes. The permeability of polyB 1 is not so high, but the good selectivity $(\alpha \simeq 4)$, and self- 
supporting property make polyB1 quite attractive as a selective oxygen permeable membrane material. In order to attain efficient permeation, thin film forming property is very important and will be studied in a future report.

To obtain more understanding of the role of poligosiloxane substituents, the permeation coefficients were separated into diffusion and solubility coefficients. Activation energies of oxygen and nitrogen permeation are also included in Table II.

The activation energy of oxygen permeation through low density polyethylene film $\left(2.8 \times 10^{-10} \mathrm{~cm}^{3} \cdot \mathrm{cm} \mathrm{cm}^{-2} \mathrm{~s}^{-1} \mathrm{~cm} \mathrm{Hg}^{-1}\right)$ is very close to reported value in the Table. ${ }^{10}$ This fact ensure the accuracy of the present data. By introducing oligosiloxanyl substituents at the $p$-position, the permeation coefficients increased 6-33 times compared with polystyrene (polySt). The change in the solubility coefficient was very small. But the diffusion coefficients increased 12-32 times, and had good correlation with the increase in $P_{\mathrm{O}_{2}}$. From above mentioned facts, it can be seen that the increase in $P_{\mathrm{O}_{2}}$ of $p$-oligosiloxanyl substituted polySt essentially depend on the increase in $D_{\mathrm{O}_{2}}$ and not on $S_{\mathrm{O}_{2}}$.

The activation energy of permeation can be separated into activation energies of diffusion and dissolution.

$$
\begin{aligned}
& D=D^{*} \exp \left(-E_{\mathrm{D}} / R T\right) \\
& S=S^{*} \exp \left(-E_{\mathrm{S}} / R T\right)
\end{aligned}
$$

From eq $5-7$, eq 8 is obtained.

$$
E_{\mathrm{P}}=E_{\mathrm{D}}+E_{\mathrm{S}}
$$

The activation energies in the permeation of oxygen and nitrogen are rather small. Although it is difficult to discuss the differences in absolute values of activation energies at this moment, the activation energies of glassy polymers (polyS2 and polyB1) at the temperature range of measurement are quite close to that of polySt (glassy), and even very close to the activation energies of the diffusion through PDMS $\left(2-3 \mathrm{kcal} \mathrm{mol}^{-1}\right){ }^{11}$ Barrie $^{13}$ studied the permeation of gaseous hydrocarbons through the block and graft copolymers of PDMS and polySt, and reported the activation energy $E_{\mathrm{D}}=3.33 \mathrm{kcal}$ $\mathrm{mol}^{-1}$ for the permeation of methane through PDMS. Since it is recognized that $E_{\mathrm{S}}$ is about $2 \mathrm{kcal} \mathrm{mol}^{-1}$, the values indicate that there are not so many differences in the activation energies between polySn and polySt or PDMS. This fact might indicate that the introduction of $p$-oligosiloxanyl substituents at the $p$-position of polySt does not change the activation energies of diffusion and solubilization so much. Since, as discussed earlier, the increase of $P_{\mathrm{O}_{2}}$ completely depends on the increase of $D_{\mathrm{O}_{2}}$ and not on $S_{\mathrm{O}_{2}}$, and the change of activation energies by introduction of $p$-substituents is quite small, the increase of $P_{\mathrm{O}_{2}}$ seems to be basically controlled by the increase of $D^{*}$ frequency factor in diffusion.

The $D^{*}$ factor is considered as a measure of the density and size of free volumes. ${ }^{14,15}$ Accordingly, by introducing $p$-substituents into polySt, the free volumes are considered to have increased. PolyS2 has a 6 times higher permeability than polyBS which has alkyl side chains similar in size with polyS2. PolyB 1 shows 2 times higher $P_{\mathrm{O}_{2}}$ than polyBS. These facts cleanly indicate the importance and role of $p$-oligosiloxanyl substituents in enhancing the permeability of gases through the increase in number and diffusibility of free volumes. These effects are considered to be closely related to the flexibility of oligosiloxanyl chains compared with alkyl chains.

Further detailed studies are needed to obtain more precise insights into permeation.

Acknowledgement. Financial support by a Grant-in-Aid for Scientific Research (No. 60550639) from Ministry of Education, Science and Culture, and Ishida Science Foundation is gratefully acknowledged. 


\section{REFERENCES}

1. Y. Kawakami, T. Aoki, H. Hisada, Y. Yamamura, and Y. Yamashita, Polym. Comm., 26, 133 (1985).

2. Y. Kawakami, H. Karasawa, T. Aoki, Y. Yamamura, H. Hisada, and Y. Yamashita, Polym. J., 17, 1159 (1985).

3. R. Adams and C. R. Noller, Org. Synth., Coll. Vol. I, 109 (1939).

4. Huang-Minlon, J. Am. Chem. Soc., 68, 2487 (1946), 71, 3301 (1949).

5. D. Todd, Org. React., 4, 378 (1948).

6. K. Tamao, K. Sumitani, Y. Kiso, M. Zenbayashi, A. Fujioka, I. Nakajima, A. Minato, and M. Kumada, Bull. Chem. Soc. Jpn., 49, 1958 (1976).

7. H. A. Daynes, Proc. R. Soc. London, Ser. A, 97, 286 (1920).
8. R. M. Barrer, Trans. Faraday Soc., 35, 628, 644 (1939).

9. W. A. Rogers, R. S. Burity, and D. Alpert, J. Appl. Phys., 25, 868 (1954).

10. A. S. Michaels and H. J. Bixler, J. Polym. Sci., 50, 413 (1961).

11. R. M. Bawer and H. T. Chio, J. Polym. Sci., C, 10, 111 (1966).

12. T. Nakagawa, "Makugaku Nyuumon," Kitami Syobou, Tokyo, 1978, p 273.

13. J. A. Barrie and K. Munday, J. Membrane Sci., 13, 175 (1983).

14. K. D. Ziegel and F. R. Eirich, J. Polym. Sci., A-2, 8, 2015 (1970).

15. H. Fujita, A. Kishimoto, and K. Matsumoto, Trans. Faraday Soc., 56, 424 (1960). 